\title{
UNIVERSITYOF
}

FORWARD

THINKING

WESTMINSTER用

WestminsterResearch

http://www.westminster.ac.uk/westminsterresearch

\section{Evaluating Socioeconomic Influences on Pinterest Consumer}

Behavior

Schmidt, C.A. and Evans, R.D.

This is a copy of the author's accepted version of a paper subsequently published in the proceedings of the IEEE Technology and Engineering Management Conference, Chicago, USA 28 Jun - 01 Jul 2018.

The final published version will be available online at:

https://dx.doi.org/10.1109/TEMSCON.2018.8488427

(C) 2018 IEEE . Personal use of this material is permitted. Permission from IEEE must be obtained for all other uses, in any current or future media, including reprinting/republishing this material for advertising or promotional purposes, creating new collective works, for resale or redistribution to servers or lists, or reuse of any copyrighted component of this work in other works.

The WestminsterResearch online digital archive at the University of Westminster aims to make the research output of the University available to a wider audience. Copyright and Moral Rights remain with the authors and/or copyright owners.

Whilst further distribution of specific materials from within this archive is forbidden, you may freely distribute the URL of WestminsterResearch: ((http://westminsterresearch.wmin.ac.uk/)).

In case of abuse or copyright appearing without permission e-mail repository@westminster.ac.uk 


\title{
Evaluating Socioeconomic Influences on Pinterest Consumer Behavior
}

\author{
Courtney Anne Schmidt and Richard David Evans \\ Westminster Business School \\ University of Westminster \\ London, United Kingdom \\ w1619591,R.Evans@westminster.ac.uk
}

\begin{abstract}
Given the increasing popularity of social media channels for influential consumption mechanisms, this research aims to evaluate socioeconomic factors with Pinterest behaviors, underpinned by consumer behavior characteristics. Strategic recommendations are also proposed for marketing managers to optimize their usage of Pinterest. An online questionnaire was completed by a sample of 50 Pinterest users, while 4 face-to-face interviews were conducted with the aim of providing a framework for enhanced use of Pinterest. Findings indicated that virtual exploration is seen as a key Pinterest motivator, supported by the top pinboard rankings of Food, DIY, and Home. Secondly, occupation and family status were the two most significant socioeconomic factors that influences behavioral uses of Pinterest. A concentrated population between ages 25-34, representing higher income households and occupations consisting of Teachers and Business Professionals, exemplified the highest usage and self-efficacy of Pinterest. Low frequency of actual consumption through Pinterest, resulting in high visual consumption, curation of pins, and pinning behaviors were apparent throughout all socioeconomic factors, however the minimal actual consumption can be improved upon, as ease-of-use features are enhanced to embedded e-commerce and online blogs.
\end{abstract}

Keywords-Pinterest, Socioeconomic Influences, Online Consumer Behavior, Virtual Exploration, Visual Consumption.

\section{INTRODUCTION}

The rising popularity and advancement in capabilities of Pinterest has encouraged researchers to examine varying objectives in its use, including consumer behavior $[1,2]$, motivations [3] and influential characteristics [3, 4], to illustrate the purposeful use of Pinterest. Pinterest allows users to collect, organize and share image-based content among their online community, representing their personal interests [4], tastes and inspirations [1], as well as providing an infrastructure to discover, collect, collaborate and publish [5]. Furthermore, Pinterest provides a means for consumers and marketers to identify trending themes [3], especially given an estimated two thirds of pins are related to specific brands or products [2]. One study [3] examined user preferences to use image-based social media for consumer related activities, rather than social media sites, where the emphasis was placed on textual content or a combination of image and text-based content.

Emerging definitions of consumer behavior, such as "a psychological state that occurs through interactive, co-creative consumer experiences with a focal agent/objective" [6] reinforces the changing environment of consumer behavior through technological developments and increased presence of social media. Brands have begun to realize the benefits of leveraging consumer engagement on social media platforms and identifying individuals' influences [7, 8], where not only can brand awareness and reputation strengthen, but also distribution of information to large audiences in a cost-effective manner is achievable [9, 10], while pursuing high volume traffic towards the brand via social media $[1,8]$.

Furthermore, popularity to examine influences and uses of Pinterest amongst researchers has risen over the past few years, particularly around electronic Word-Of-Mouth (eWOM) behaviors of "liking, sharing, following, collecting and pinning" or rather termed "social curation," resembling a digital library $[4,11,12]$. Based on recent trends in consumer behavior, focused on the use of Pinterest as the medium, this study aims to critically evaluate the influences of consumer behavior through users' socioeconomic status, with the aim of providing relevant information about key socioeconomic factors that have been neglected in past research. This study will examine two research questions, encompassing the topics of consumer behavior through Pinterest, underpinned by the influences of socioeconomic factors as well as a comparison of actual and visual consumption behaviors, thus leading to the following questions:

- Does socioeconomic status influence an individual to use Pinterest for consumption?

- Does the use of Pinterest translate into actual consumption or remain as visual consumption?

\section{RELATED WORK}

\section{A. Consumption of Pins - Visual Consumption}

Consumption, underpinned by the motivation of physically attaining a specific goal or item, encourages the consumer to closely evaluate which social media medium they prefer in order to optimize the attainment of the goal [3]. Visual consumption, on the other hand, is defined by the curation or collection of pins (images) and is an inherent feature of Pinterest, whereas actual, physical consumption of the image may not be the consumer's end-goal [3, 4]. Although a consumer's use of social media filtration in relation to 
obtaining a pre-determined goal has been proven effective, other research underpinned by the Technology Acceptance Model, as an appropriate means for e-commerce adoption, to evaluate the ease-of-use [3, 13] has argued the generation of more unplanned purchases, as compared to social media filtration for pre-determined goal related purchases [14]. $45 \%$ of the embedded websites on Pinterest are e-commerce based or blogging websites [11], while other research indicates that $93.4 \%$ of images posted are linked to external websites, including Google, Blogspot or Tumblr [15], giving Pinterest the capabilities of expanding and encouraging online consumption. Furthermore, the likelihood of a consumer using the online medium for purchasing, is directly linked and influenced by the effortlessness of the given technology or 'ease-of-use' and ultimately enhances consumer performance or productivity in the process, resulting in the ending outcome or rather obtaining the purchasing goal [2]. The following hypothesis is therefore posed:

- Visual consumption will be more frequent than actual consumption through Pinterest.

\section{B. eWOM's Influential Role on Consumer Behaviour}

The transformation of WOM to eWOM through the development of social media platforms has identified eWOM as the behavior of liking, sharing, commenting or, in the case of Pinterest, pinning [3, 9]. However, as the popularity of social media continues to grow, the influence of eWOM on consumer behavior has become widely accepted and researched [6, 9], primarily by marketers, in order to capture and interpret their targeted audience. In fact, attitude-defining consumer behavior, through an individual's trusted social network, has been argued to be more influential than traditional marketing methods, given the social communities were curated by the individuals they deem trustworthy for brand information dissemination and therefore spurring eWOM [3,7]. By sharing, or 'pinning' an image, the user voluntarily provides information to their social community of their personal brand or category-related preference and, therefore, stimulates eWOM [7]. Pinterest and Instagram, both image-based, have weaker research related to engagement or eWOM behaviors, however each have different motivations for use or gathering, sharing and disseminating information [9]. Additionally, several eWOM studies have related to key influencing factors as tie strength, homophily, trust, normative influence and informational influence [7], which is a diversion from the significantly impactful research [6] that defined cognitive, emotional and behavioral consumer engagement dimensions as a foundation for further research. It could be argued that tie strength, closeness of family and friends, as compared to acquaintances, suggests stronger influence of eWOM engagement, as well as homophily of common denominator socioeconomic characteristics [7]. Supported by past research, the following hypothesis is posed:

- If social connections within the user's community have pinned a common interest, there will be a Positive Influence for the user to re-pin the image.

\section{Socioeconomic Status Influence on Consumer Behaviour}

An individual's socioeconomic status can be defined by social class characteristics, involving their individual resources, encompassing household income, occupation, education level and family status [16]. These defined characteristics have encouraged research in the arena of marketing and e-commerce as indicators and influencers for consumer behavior, as well as more increasingly with online consumer behavior research [17]. However, gender differentials towards consumer behavior has also been extensively studied $[3,4,5,18]$, while statistical data [19] supporting Pinterest use warrants further socioeconomic analysis for consumer and Pinterest behaviors.

In addition, a narrowed demographic to only include college age students, indicated that a satisfactory outcome through using Pinterest was a desire to achieve an authentic experience, while the results led to higher personal expression and organization, as well as a sense of close-cultural identity [20]. However, some research has proposed that social distance outweighs the significance of customer characteristics towards consumer behavior influence [8]. Education and income factors studied have been mostly related to self-efficacy or an individual's technological capability to understand and use web-based tools [17] and a heightened use of social media as a consumption medium typically representing younger generations. Similarly, income, as a reflection of one's occupational status, may only initially bear influence towards online shopping until the self-efficacy has been thoroughly developed; therefore, no longer placing a high-level of influence on online consumer behavior [17]. Expanding upon past research to include other socioeconomic factors, the following two hypotheses are posed:

- Occupation positively correlates with the user's interests and pinboards; and

- Family status positively correlates with the user's interests and pinboards.

\section{METHOD}

This research adopted a mixed-method approach to obtain superior qualities of a combination method, enticing triangulation for a multiple procedural research approach to optimize the validation of results [21]. This allowed for a sequential explanatory mixed-method, where the primary data, collected in the first phase, was categorized by a quantitative method, utilizing an online questionnaire of 50 valid respondents, and further substantiated in the second phase utilizing a qualitative method derived by four semi-structured interviews to provide a more thorough understanding and explanation of consumer behavior related to the quantitative results of the first phase [22]. Facilitating the quantitative data collection, a structured survey optimizing the use of Likert Scales [23] was used, supporting a survey strategy where the researcher pre-determined the selected responses to eliminate any biases that could potentially affect the results, whilst SurveyHero.com was the facilitating tool for distribution of the online questionnaire. The quantitative data collection aimed to address the four hypotheses posed, with the subsequent qualitative data collection aimed to address the three key themes, through utilizing non-standardized, semi-structured 
interviews. The four interviewees represented four different occupations and family statuses.

Quantitative analysis was facilitated through SPSS [24] and was initially used to provide descriptive statistics to explain the sample population and limits of the data collected from the questionnaire. This was followed by Chi-Square tests to examine the existence of relationships with statistical significance between the socioeconomic factors, as the dependent variable, and the three-tiered pinboard category rankings, representing the 32 Pinterest-created pinboards, previously studied [4] as the independent variables. Furthermore, Bivariate Correlations were employed to compare the pre-defined motivations conducted in past research [3].

\section{RESULTS}

\section{A. Behavioural and Social Influence Results}

Table 1 illustrates three new variables calculated to encompass pinning behaviors, consumer behaviors and social influences. Given the means, pinning behavior not only represented the highest mean, but also the highest standard deviation, which is significantly higher than consumer behavior's mean and standard deviation. Founded upon the same Likert Scale whilst represented by the average of the five social and socioeconomic questions, the participants' perceptive level of influence and similarity amongst their social connections represented a relatively low-medium mean, as well as a low standard deviation.

TABLE I. NEW VARIABLES

\begin{tabular}{|l|c|c|c|}
\hline \multicolumn{1}{|c|}{ Variable } & Formula & Mean & S.D. \\
\hline Pinning Behavior & $\begin{array}{c}\text { = Frequency of use * } \\
\text { probability of pinning }\end{array}$ & 13.58 & 7.892 \\
\hline Consumer Behavior & $\begin{array}{c}\text { = Probability of clicking } \\
\text { link } * \text { purchasing probability }\end{array}$ & 6.78 & 3.699 \\
\hline Social Influence & $\begin{array}{c}\text { avg. social similarities and } \\
\text { socioeconomic statuses }\end{array}$ & 2.12 & 0.587 \\
\hline
\end{tabular}

\section{B. Pinboard Ranking and Socioeconomic Results}

Through the use of Chi-Square testing on all three pinboard tiers and individual socioeconomic factors, statistical significances were determined $(p<0.05)$, however not all individual socioeconomic levels were deemed significant.
Those who were "in a relationship" showed a significant association for Women's Style in the second tier and Education in the third tier, whilst the "engaged" population associated with Design in the first tier and Weddings in the second tier for pinboard rankings. Although more than half (56\%) was either "married" or "married with child(ren),"; there were no significant associations detected within the three-tier ranking.

However, nearly all occupations indicated some level of significant associations with at least one tier in the rankings. Business professionals, representing $32 \%$ of the sample, indicated the same significant association levels for Women's Style in the first and second tier. Additionally, Humor and Travel were associated on the second and third levels, respectively. Similarly, Teachers, representing $26 \%$ of the sample, indicated significant association levels for Education in both the first and second tiers, whilst the Kids pinboard also proved significant in the second tier, yet slightly less significant than Education.

Overall, the first tier's responses represented 13 different pinboards, whilst the second and third tier responses contained 15 and 19, respectively. The first tier was more concentrated around the Food pinboard $(\mathrm{N}=22,44 \%)$, whereas the second and third tiers were diversified between Do-It-Yourself (DIY), $(\mathrm{N}=10,20 \%)$ and Home $(\mathrm{N}=8,16 \%)$, accordingly. The Food pinboard was the only pinboard that was either the top or second highest pinboard within each tier consistently, whereas Home, Holiday \& Party, and Kids became higher ranked as the tiers increased.

\section{Proposed Framework}

Figure 1 represents the proposed research framework, as an extension of not only the Uses and Gratification (U\&G) model, where underlying motivations and engagements of consumption through social media are examined, but also a past Pinterest study [3] to include the pre-determined motivations and additional behavioral and socioeconomic variables, as identified in Section A of the results. Similarly, bivariate correlations amongst the motivations were performed to explain the associated degree of relationship between two variables, indicating significant correlation (significant at the .01 level; 2-tailed) between virtual exploration and cuisine $(\mathrm{r}=$ $.367, \mathrm{n}=50, \mathrm{p}=.009, \mathrm{p}<.01)$, and between cuisine and creative projects $(\mathrm{r}=-.391, \mathrm{n}=50, \mathrm{p}=.005, \mathrm{p}<.001)$. 


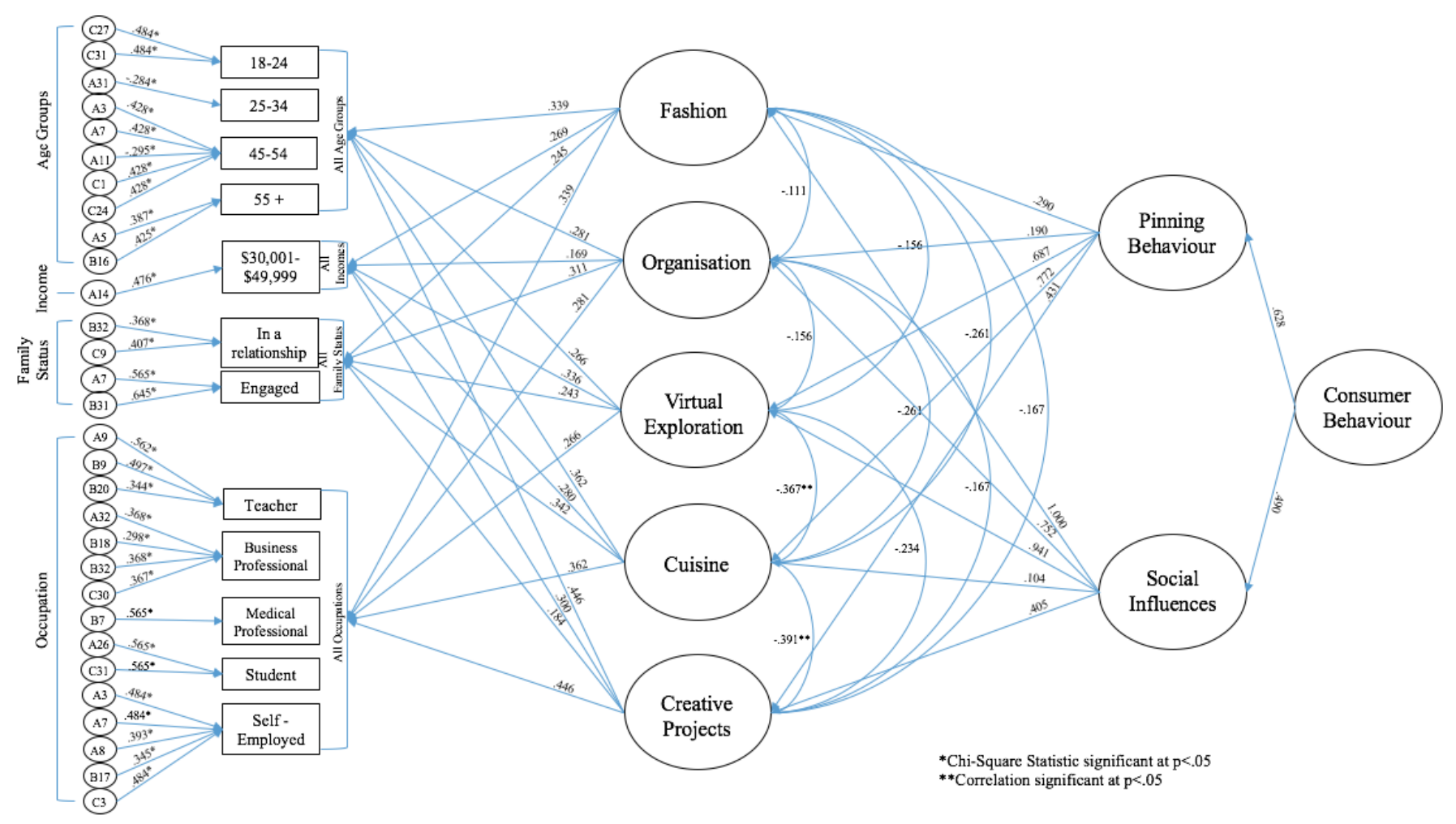

Fig. 1. Proposed Research Framework

\section{DISCUSSION}

\section{A. Visual Consumption will be more Frequent than Actual Consumption through Pinterest}

Quantitatively, 80\% ( $\mathrm{n}=40)$ of respondents indicated that they would never or almost never purchase through Pinterest, whereas the remaining $20 \%(n=10)$ would only occasionally purchase through Pinterest, leading to accepting or failure to reject this hypothesis of visual consumption occurring more often than actual consumption through Pinterest. Additionally, the higher mean of Pinning Behavior compared to Consumer Behavior variable supports the accepting of the hypothesis.

However, qualitatively, the interviewees' indicated some indifference, where half of the interviewees had a tendency to make actual purchases inspired by their curated pins, yet the difference in actual purchases lie amongst the vehicle of either online or in-store, dependent upon the purchasing item in question for not only quality purposes, but also preference of the experiential-orientated consumer [25, 26]. Some [27] argue that the use of multichannel evaluation for consumption can vary dependent on the phase in which the consumer is at in their decision-making process and information gathering process, leading to exploration (similar to visual consumption for a future purchase), online purchases and in-store purchases.

\section{B. If Social Connections within a User's Community have pinned a common interest, there will be a Positive Influence for the user to re-pin the image}

The perception of influence of similar social status towards re-pinning ranged from not influential at all $(36 \%, \mathrm{n}=18)$ to somewhat influential $(38 \%, n=19)$, whilst $86 \%(n=43)$ viewed their socioeconomic status similarity compared to their peers as either somewhat or very similar. The Chi-Square test between the similarity of a user's socioeconomic status similarity with their social connections compared to their influence of repinning provided no statistical significance and, therefore, fails to accept this hypothesis.

Qualitative analysis provided clarification where 3/4 indicated that their social connections are similar socioeconomically, yet there is not a significant influence towards re-pinning the same images, similar to a prior study [4] where pin characteristics were viewed more influential than social characteristic similarities, whilst opposing another study's results [7] where relational tie strength encouraged more eWOM behavioral characteristics. However, notice was taken of similar trends with their peers' pinning behavior, specifically in the DIY, Home, Education and Kids pinboards, which inherently some correlated with the interviewee's shared occupation (e.g. teacher) or family status (e.g. married with a child). Therefore, despite the quantitative rejection of this hypothesis, qualitative findings indicate there is some influential relationship or rather shared categorical inspirations 
amongst those users with similar socioeconomic statuses. However, the re-pinning influence is not significant, supporting the informational behaviors driven by knowledge gathering through observations of online peers, as compared to normative behaviors $[7,28]$.

\section{Occupation Positively correlates with the User's Interests and Pinboards}

In the proposed research framework, statistical significance indicated the highest quantity of pinboard rankings amongst the business professionals and self-employed individuals, however there were more first-tier pinboards (i.e. Art, Design, DIY) for self-employed individuals, where business professionals only had one first-tier variable of Women's Fashion. Additionally, teachers demonstrated significance for the Education pinboard in both the first and second-tiers, whilst the Kids pinboard was significant at the second-tier level, as these categories had direct relationships with their occupation. While the separation of individual occupations to the pinboard rankings, proved significance in certain pinboards across the three tiers, not all directly related to the occupational characteristics. However, taking into consideration all occupations as a unified variable and the individual motivations examined in this study, there was no statistical significance between the variables, therefore rejecting the hypothesis based on the quantitative analysis.

Congruently, the qualitative interviews indicated a weak relationship between the interviewees' occupational status and influence on their first-tier ranking, where three-fourths preferred Food, whilst the other indicated Education, as it directly related to her occupation (i.e. teacher). Subsequently, the second and third-tiers elaborated on more creative-based boards, including DIY, Design and Beauty, where the underlying motivation was virtual exploration with the goal of obtaining inspirational content. The third-tier rankings varied across Women's Style, Design, Photography and Home, where only Women's Style and Business Professional showed statistical significance in the research framework, but not on a third-tier level, ultimately leading to the agreement of the quantitative findings to reject this hypothesis.

\section{Family Status positively correlates with the User's interests and pinboards}

The research framework generated in this study elicits two family statuses, "in a relationship" and "engaged" as holding a statistical significance each with two variables of pinboard rankings. Those "in a relationship" showed significance in the second and third-tiers, representing Women's Style and Education respectively, whilst those that were "engaged" showed significance in the first and second-tiers, representing Design and Weddings, which could arguably be related to interests and characteristics associated with an engaged individual. Given only one-third of the family statuses listed were statistically significant, a rejection of this hypothesis could be warranted on a quantitative analysis basis. Yet, through qualitative analysis, the clarity of family status' influence towards pinning behavior was very relevant for three interviewees, with the most significant one relating nearly all her motivations for Pinterest usage and motivations driven by exploration for her child and home, which she had recently purchased with her husband. Furthermore, interviewees with both "single" and "in a relationship" statuses did not see a direct influence of their family status towards their own pinning behaviors, however, both interviewees noticed similar pinning behaviors amongst their peers with similar family status, specifically those "married" and "married with children". In fact, these two interviewees also found it interesting to explore what pins they had curated and find similar patterns to their own interests, despite having different family statuses. However, it has been argued [9] that homophily influence discourages access to a diverse range of information and knowledge, therefore encouraging similar interest amongst those with indicating homophily and thus an argument could be warranted to accept this hypothesis, as family status through the qualitative findings repeatedly enticed a strong influential relationship.

\section{CONCLUSION}

Since its inception in 2010, Pinterest has not only rapidly grown in popularity, but also increased interest for academics and researchers alike, as it serves as a strong medium to evaluate consumption behaviors, eWOM and social curation behaviors, as well as demographic characteristics of Pinterest users. Yet, a gap was identified in past studies where a more comprehensive view of potential influential socioeconomic factors was neglected, in relation to Pinterest and consumer behavioral traits. This research aimed to answer not only the socioeconomic influences for Pinterest-inspired consumption, but also provide a comparison of actual versus visual consumption use of Pinterest.

Expanding upon a past Pinterest-based research framework [3], was the inclusiveness of additional intervening variables, whilst incorporating the already pre-determined exploratory Pinterest motivations. This study identified socioeconomic factors with the supporting statistical significant pinboard rankings, whilst structuring the variables' relationships with motivations, pinning behaviors and social influences, and ultimately illustrated the overall consumer behavior through Pinterest. The triangulation of the research methodology allowed for the critical findings extending into a discussion to assess the four hypotheses amongst both the quantitative and qualitative findings, all of which were founded upon the three key themes of consumption of pins, influence of eWOM and socioeconomic influences.

As the sample population concentrated on the Midwest United States only, an expansion of geographical regions is suggested to not only increase the sample population size and thus increasing the likelihood of data reliability, but also to provide a stronger criticality of regional differences in consumer and Pinterest behaviors and their underlying socioeconomic influences through the optimization of this study's proposed research framework.

\section{ACKNOWLEDGMENT}

The authors would like to thank all those who voluntarily participated in the online questionnaire and those that participated in the subsequent interviews. 


\section{REFERENCES}

[1] Youn, S. and S. Jin, "Reconnecting with the past in social media: The moderating role of social influence in nostalgia marketing on Pinterest" Journal of Consumer Behaviour, vol. 16, no. 6, pp. 565-576, 2017.

[2] Perea, T., B. Dellaert and K. Ruyter, "What drives consumers to shop online? A literature review" International Journal of Service Industry Management, vol. 15, no. 1, pp. 102-121, 2004

[3] Mull, L. and S. Lee, “ "PIN" Pointing the motivational dimensions behind Pinterest" Computers in Human Behavior, vol. 33, pp. 192-200, 2014.

[4] Han, J., D. Choi, B. Chun, T. Kwon, H. Kim and Y. Choi, "Collecting, organizing, and sharing Pins in Pinterest: Interest-driven or socialdriven?" in International Conference on Measurement and Modeling of Computer Systems, New York, USA, 2014, pp. 15-27.

[5] Zarro, M., C. Hall and A. Forte, "Wedding dresses and wanted criminals: Pinterest.com as an infrastructure for repository building" in International AAAI Conference on Weblogs and Social Media, Boston, USA, 2013, pp. 650-658.

[6] Brodie, R., L. Hollebeek, B. Jurić and A. Ilić, "Customer engagement: Conceptual domain, fundamental propositions, and implications for research" Journal of Service Research, vol. 14, no. 3, pp. 252-271, 2011.

[7] Chu, S. and Y. Kim, "Determinants of consumer engagement in electronic word-of-mouth (eWOM) in social networking sites" International Journal of Advertising, vol. 30, no. 1, pp. 47-75, 2011.

[8] Libai, B., R. Bolton, M.S. Bügel, K. de Ruyter, O. Götz, H. Risselada, A.T. Stephen, "Customer-to-customer interactions: Broadening the scope of word of mouth research" Journal of Service Research, vol. 13, no. 3, pp. 267-282, 2010.

[9] Dessart, L., C. Veloutsou and A. Morgan-Thomas, "Consumer engagement in online brand communities: a social media perspective" Journal of Product \& Brand Management, vol. 24, no. 1, pp. 28-42, 2015.

[10] Godey, B., A. Manthiou, D. Pederzoli, J. Rokka, G. Aiello, R. Donvito and R. Singh, "Social media marketing efforts of luxury brands: Influence on brand equity and consumer behaviour" Journal of Business Research, vol. 69, no. 12, pp. 5833-5841, 2016.

[11] Hall, C. and M. Zarro, "Social Curation on the Website pinterest.com" Proceedings of the Association for Information Science and Technology, vol. 49, no. 1, pp. 1-9, 2012.

[12] Chang, S., V. Kumar, E. Gilbert and L.G. Terveen, "Specialization, homophily, and gender in a social curation site: Findings from Pinterest" in ACM Conference on Computer Supported Cooperative Work \& Social Computing, Baltimore, USA, 2014, pp. 674-686.

[13] Jin, Y., J. Lin, B. Gilbreath and Y. Lee, "Motivations, consumption emotions, and temporal orientations in social media use: A strategic approach to engaging stakeholders across platforms" International Journal of Strategic Communication, vol. 11, no. 2, pp. 115-132, 2017.

[14] Koufaris, M., "Applying the technology acceptance model and flow theory to online consumer behaviour" Information Systems Research, vol 13, no. 2, pp. 205-223, 2002.

[15] Feng, Z., F. Cong, K. Chen and Y. Yu, "An empirical study of user behaviors on Pinterest social network" in IEEE/WIC/ACM International Joint Conferences on Web Intelligence (WI) and Intelligent Agent Technology (IAT), Atlanta, USA, 2013, pp. 402-409.

[16] Shavitt, S., D. Jiang and H. Cho, "Stratification and segmentation: Social class in consumer behaviour" Journal of Consumer Psychology, vol. 26, no. 4, pp. 583-593, 2016.

[17] Hernández, B., J. Jiménez and M.J. Martin, “Age, gender and income: do they really moderate online shopping behaviour?" Online Information Review, vol. 35, no. 1, pp. 113-133, 2011.

[18] Ottoni, R., J.P. Pesce, D.L. Casas, G. Franciscani Jr., W. Meira Jr., P. Kumaraguru and V. Almeida, "Ladies First: Analyzing gender roles and behaviors in Pinterest" in International AAAI Conference on Weblogs and Social Media, Ann Arbor, USA, 2013, pp. 457-465.

[19] Greenwood, S., A. Perrin and M. Duggan, "Social media update 2016" in Pew Research Center: Internet \& Technology, 2016.

[20] Sashittal, H. and A. Jassawalla, "Why do college students use Pinterest? A model and implications for scholars and marketers" Journal of Interactive Advertising, vol. 15, no. 1, pp. 54-66, 2015.

[21] Johnson, R.B., A. Onwuegbuzie and L. Turner, "Toward a definition of mixed methods research" Journal of Mixed Methods Research, vol. 1, no. 2, pp. 112-133, 2007.

[22] Saunders, M., Research Methods for Business Students. Pearson Education Limited, 2016.

[23] Ekinci, Y., Designing Research Questionnaires. SAGE Publications, 2015.

[24] Dawson, J., Analysing Quantitative Survey Data for Business and Management Students. SAGE Publications, 2017.

[25] Li, H., C. Kuo and M. Rusell, "The impact of perceived channel utilities, shopping orientations, and demographics on the consumer's online buying behaviour" Journal of Computer-Mediated Communication, vol. 5, no. 2, pp. 0, 1999.

[26] Burke, R., "Technology and the customer interface: What customers want in the physical and virtual store" Journal of the Academy of Marketing Science, vol. 30, no. 4, pp. 411-432, 2002.

[27] Chiou, J., S. Chou and G. Shen, "Consumer choice of multichannel shopping: The effects of relationship investment and online store preference” Internet Research, vol. 27, no. 1, pp. 2-20, 2017.

[28] Wang, D., Z. Wen, H. Tong, C. Lin, C. Song and A. Barabási, "Information spreading in context" in International Conference on World Wide Web, Hyderabad, India, 2011, pp. 735-744. 